\title{
Quality of Services Provisioning in Wireless Sensor Networks using Artificial Neural Network: A Survey
}

\author{
Mohit Mittal \\ Research Scholar \\ Department of computer science \\ Gurukul Kangri University \\ Haridwar, India
}

\author{
Krishan Kumar \\ Assistant Professor \\ Department of computer science \\ Gurukul Kangri University \\ Haridwar, India
}

\begin{abstract}
Wireless sensor network (WSN) is one of emerging trends in networking technologies being used for communication purpose in modern life. It has mainly comprised of small sensor nodes (SNs) with limited resources. Individual SNs are connected with each other and make the communication possible. Enhancement in the communication among sensor nodes or Sensor-to-Sink nodes is today's most prominent objective. In this paper we have surveyed artificial neural network for different QOS parameters of WSN. Artificial neural network (ANN) is very prominent emerging area for WSN applications. Generally, artificial neural networks are classified in supervised learning and unsupervised learning. Unsupervised learning includes algorithms like Hebbian, Winner-take-all, ART, ART1, ART2, counter propagation network etc., while supervised learning includes perceptron model, delta learning rule, error back-propagation etc. ANN helps to achieve the better quality of services for communication in wireless sensor networks at the greater extent. We have summarized the survey of neural networks' techniques applied for WSN applications so far.
\end{abstract}

\section{Keywords}

Wireless sensor network; artificial neural network; unsupervised learning; supervised learning; Fuzzy ART; ART1; ART2; perceptron model; error back propagation; quality of services.

\section{INTRODUCTION}

Wireless Sensor Network is formed by groups of small sensor nodes (SNs) which are deployed for various collaborative missions such as environmental monitoring, target tracking and surveillance. As due to the miniature size of the sensor nodes, they are typically deployed in large numbers, and communicate via multiple hops through a wireless shared communication channel. The proper implementation of wireless sensor network is generally dependent on the enabling technologies such as digital electronics and wireless communications, as well as the provisioning of Quality of Service (QoS) in the network. In traditional networks; Quality of Service mechanism provides the service parameter such as delay, bandwidth, and packets lost rate etc. QoS is defined as the network's promise for the quality of user's data transmission service. It can be classifiable for different grade of service and provide different transport capabilities. In the wireless sensor networks (WSN), the nodes of the network are not only to transmit data, but also need to take up the task of monitor the environment. The efficiency of its applications does not only rely upon the transmission ability but also the monitor ability. So, the QoS of the WSN rely on the specific application, such as the monitor ability of events, the covered area of network, the energy consumption of network [40].

The typical application of wireless sensor network is event monitoring. WSN need not only to monitor the temperature and humidity of the forests but also used to detect the fire at different places in the forest. If; the fire is detected, the data of event must be transmitted rapidly. This event requests high real-time performance and the WSN must have the QoS mechanism to satisfy the different grade of service. The research of WSN's QoS is focused on the end-to-end realtime and reliability. When the event occurred, it can be detected and transmitted rapidly in with low-energy consumption. The QoS mechanism should improve the efficiency and reduce the energy consumption of sensor nodes to delay the network's survival time. The improved mechanism is fit for the WSN with limited resources and it has the advantages such as less energy consumption, less computation amounts, less communication data etc [40].

WSN is complex network which consists of sensing, processing and communication. It is driven by various applications and highly requires new Quality of Service guarantees. The application target of WSN is to monitor events, and it pays more attention to the QoS of colony data packets. The QoS mechanism considers the different event delay requirements, and adjusts the bandwidth and event delay reasonably for each event [18].

\subsection{Applications}

Wireless sensor networks are deployed in different fields. There is very wide range of applications from which some of are mentioned in table I

TABLE I. Summary of application based wireless sensor networks

\begin{tabular}{|c|c|c|c|}
\hline $\begin{array}{l}\text { S. } \\
\text { No. }\end{array}$ & Sensor & $\begin{array}{l}\text { Application } \\
\text { area }\end{array}$ & Sensed event \\
\hline 1. & Accelerometer & $\begin{array}{l}\text { AVM, SHM, } \\
\text { Health care, } \\
\text { Transportation }\end{array}$ & $\begin{array}{l}2 \mathrm{D} \text { and } 3 \mathrm{D} \text { acceleration of } \\
\text { movements of people and } \\
\text { objects }\end{array}$ \\
\hline 2. & $\begin{array}{l}\text { Acoustic emission } \\
\text { sensor }\end{array}$ & SHM & $\begin{array}{l}\text { Elastic waves generated } \\
\text { by the energy released } \\
\text { during propagation }\end{array}$ \\
\hline & Acoustic sensor & $\begin{array}{l}\text { Transportation } \\
\text { Pipelines }\end{array}$ & $\begin{array}{l}\text { Acoustic pressure } \\
\text { vibration }\end{array}$ \\
\hline 3. & $\begin{array}{l}\text { Capacitance } \\
\text { sensor }\end{array}$ & PA & Solute concentration \\
\hline 4. & ECG & Health care & Heart rate \\
\hline 5 . & EEG & & Brain electrical activity \\
\hline 6. & EMG & & Muscle activity \\
\hline 7. & $\begin{array}{l}\text { Electrical/electro- } \\
\text { magnetic sensors }\end{array}$ & PA & $\begin{array}{l}\text { Electrical } \\
\text { resistivity/conductivity } \\
\text { capacitance or inductance } \\
\text { affected by the } \\
\text { composition of tested soil }\end{array}$ \\
\hline
\end{tabular}




\begin{tabular}{|c|c|c|c|}
\hline 8. & Gyroscope & Health care & Angular velocity \\
\hline 9. & Humidity sensor & $\begin{array}{l}\mathrm{PA} \\
\mathrm{HM}\end{array}$ & $\begin{array}{l}\text { Relative as well as } \\
\text { absolute humidity }\end{array}$ \\
\hline 10. & Infrasonic sensor & AVM & $\begin{array}{l}\text { Concussive acoustic } \\
\text { waves produced as a } \\
\text { result of earth quake or } \\
\text { volcanic eruption }\end{array}$ \\
\hline 11. & Magnetic sensor & Transportation & $\begin{array}{l}\text { Presence intensity, } \\
\text { direction, rotation, and } \\
\text { variation of magnetic field }\end{array}$ \\
\hline 12. & Oximeter & Health care & $\begin{array}{l}\text { Blood oxygenation of } \\
\text { patients hemoglobin }\end{array}$ \\
\hline 13. & $\mathrm{pH}$ sensor & $\begin{array}{l}\begin{array}{l}\text { Pipeline } \\
\text { (water) }\end{array}\end{array}$ & $\begin{array}{l}\text { Concentration of } \\
\text { hydrogen ions }\end{array}$ \\
\hline 14. & $\begin{array}{l}\text { Photo acoustic } \\
\text { spectroscopy }\end{array}$ & Pipeline & Gas sensing \\
\hline 15. & $\begin{array}{l}\text { Piezoelectric } \\
\text { cylinder }\end{array}$ & Pipeline & Gas velocity \\
\hline 16. & $\begin{array}{l}\text { Soil moisture } \\
\text { sensor }\end{array}$ & PA & Soil moisture \\
\hline 17. & $\begin{array}{l}\text { Temperature } \\
\text { sensor }\end{array}$ & $\begin{array}{l}\text { PA } \\
\text { HM }\end{array}$ & Temperature \\
\hline 18. & Barometer sensor & $\begin{array}{l}\mathrm{PA} \\
\mathrm{HM}\end{array}$ & $\begin{array}{l}\text { Pressure exerted on a } \\
\text { fluid }\end{array}$ \\
\hline 19. & $\begin{array}{l}\text { Passive infrared } \\
\text { sensor }\end{array}$ & $\begin{array}{l}\text { Health care } \\
\text { HM }\end{array}$ & $\begin{array}{l}\text { Infrared radiation from } \\
\text { objects }\end{array}$ \\
\hline 20. & Seismic sensor & AVM & $\begin{array}{l}\text { Measure primary and } \\
\text { secondary seismic } \\
\text { wave(body wave, ambient } \\
\text { vibration) }\end{array}$ \\
\hline 21. & Oxygen sensor & Health care & $\begin{array}{l}\text { Amount and proportion of } \\
\text { oxygen in the blood }\end{array}$ \\
\hline 22. & Blood flow sensor & Health care & $\begin{array}{l}\text { The Doppler shift of a } \\
\text { reflected ultrasonic wave } \\
\text { in the blood }\end{array}$ \\
\hline
\end{tabular}

\section{ARTIFICIAL NEURAL NETWORK}

The human brain, which possesses an extraordinary ability to learn, memorize and generalize, is a dense network of over 10 billion neurons, each connected on average to about 10,000 other neurons. Each neuron receives signals through synapses, which control the effects of the signals on the neuron. These synaptic connections play an important role in the behavior of the brain. These findings have inspired modeling of biological neural systems by means of NNs [31] [45].

The three basic components of an artificial neuron shown in

Figure 1 are:

1) The links that provide weights $W j i$, to the $n$ inputs of $j$ th neuron $x i, i=1, \ldots, n$;

2) An aggregation function that sums the weighted inputs to compute the input to the activation function

$$
u j=\Theta j+\sum_{i=1}^{n} x i W j i
$$

Where $\Theta j$ is the bias, which is a numerical value associated with the neuron. It is convenient to think of the bias as the weight for an input $x 0$ whose value is always equal to one, so that

$$
u j=\sum_{i=0}^{n} x i \text { Wji }
$$

3) An activation function $\Psi$ that maps $u j$ to $v j=\Psi(u j)$, the output value of the neuron. Some examples of the activation functions are: step, sigmoid, tan hyperbolic and Gaussian function [31].

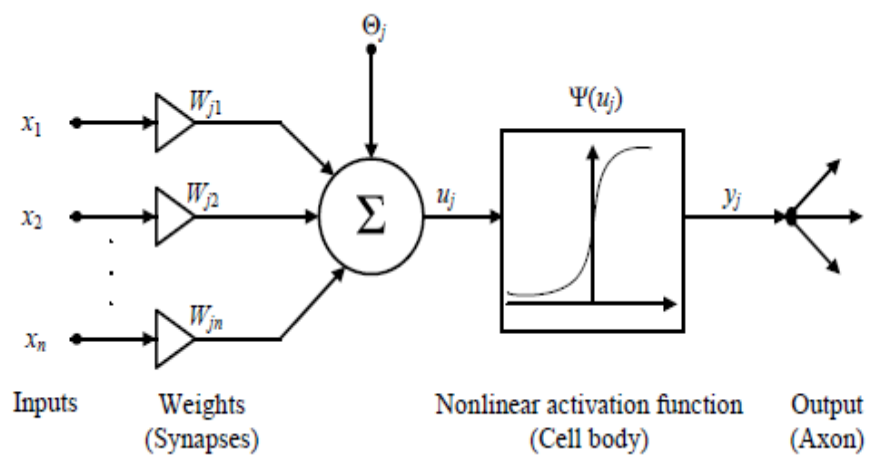

Fig. 1 Structure of an artificial neuron [31]

Neural networks are made of basic units arranged in layers. A unit collects information provided by other units (or by the external world) to which it is connected with weighted connections called synapses. These weights, called synaptic weights multiply (i.e., amplify or attenuate) the input information. A positive weight is considered excitatory, while a negative weight is inhibitory [52].

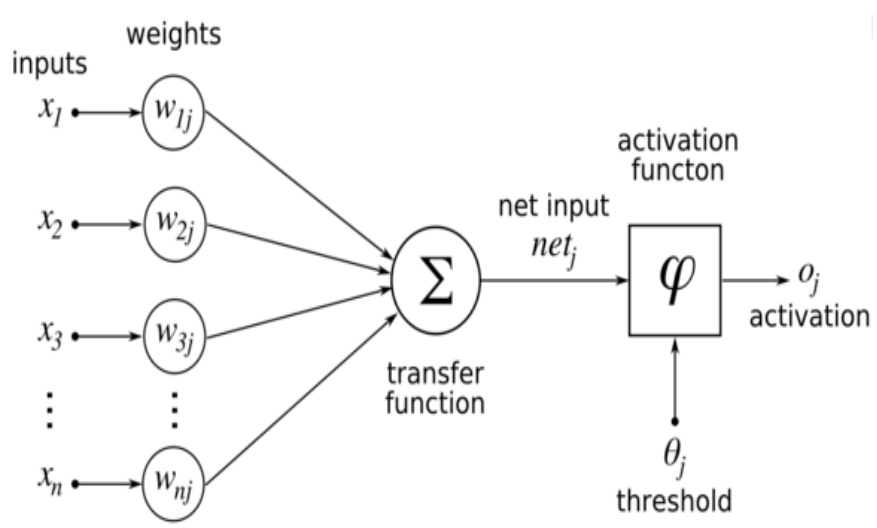

Fig.2 basic neural network models

Each of these units is a simplified model of a neuron and transforms its input information into an output response. This transformation involves two steps: First, the activation of the neuron is computed as the weighted sum of it inputs, and second this activation is transformed into a response by using a transfer function [52].

Neural networks learn the facts represented by patterns and determine their inter-relationships. Learning is the process in which the weights of a $\mathrm{NN}$ are updated in order to discover patterns or features in the input data. Learning methods are generally classified into the two types: i) supervised learning and ii) unsupervised learning. In supervised learning, a teacher presents an input pattern and the corresponding target output. Network weights are adapted in such a way that the error is minimized. The objective of unsupervised learning is to discover patterns in the input data with no help from a teacher [31].

\subsection{Unsupervised Learning}

This is also known as competitive learning. During unsupervised learning ANN typically perform dimensionality reduction or pattern clustering. They are able to discover both 
regularities and irregularities in the redundant input data by iterative process of adjusting weights of interconnections between a large numbers of simple computational units (called artificial neurons) [30].

\subsubsection{Hebbian learning rule}

The earliest and simplest learning rule for an unsupervised learning neural network is generally known as the Hebb rule [19]. Hebb proposed that learning occurs by modification of the synapse strength (weight) in a manner such that if two interconnected neurons are both "on" at the same time, then the weight between those neurons should be increased. The original statement only talks about neurons firing at the same time. However, a stronger form of learning occurs if we also increase the weights are both neurons "off" at the same time [33]. Hebbian learning rule state that the learning signal is equal to the neuron's output. We have

$$
\mathrm{r} \equiv f\left(\mathrm{w}_{\mathrm{i}}^{\mathrm{t}} \mathrm{x}\right)
$$

Where $\mathrm{r}$ is the learning signal, $\mathrm{x}$ is the input vector, $\& \mathrm{w}$ is the weight vector. Moreover the increment of the weight vector becomes

$$
\Delta \mathrm{w}_{\mathrm{i}}=\mathrm{c} f\left(\mathrm{w}_{\mathrm{i}}^{\mathrm{t}} \mathrm{x}\right) \mathrm{x}
$$

This learning rule requires the weight initialization at small random values around $\mathrm{w}_{\mathrm{i}}=0$ prior to learning. The Hebbian learning rule represents a purely feed-forward, unsupervised learning neural network. The rule states that if the cross product of output and input, or correlation term $o_{i} x_{j}$ is positive, this results in increase of weight $\mathrm{w}_{i j}$; otherwise the weight decreases [57].

\subsubsection{Winner-take-all}

It can only be demonstrated and explained for an ensemble of neurons, preferably arranged in a layer of $p$ units. This rule is an example of competitive learning, and it is used for learning statistical properties of inputs. The learning is based on the premise that one of the neurons in the layer, say $\mathrm{m}^{\text {th }}$ has the maximum response due to input $\mathrm{x}$, as shown in figure. This neuron is declared as the winner [57]. As a result of this winning event, the weight vector $\mathrm{w}_{\mathrm{m}}$ becomes:

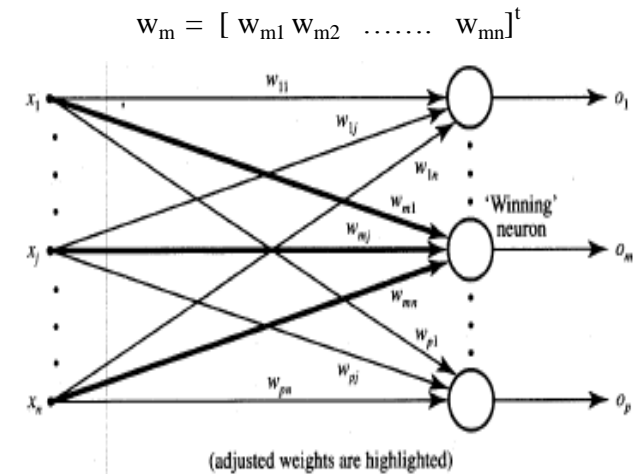

Fig.3 Basic model of winner takes all learning rule [57]

\subsubsection{Adaptive Resonance Theory (ART)}

Adaptive resonance theory (ART) was developed by Carpenter and Grossberg in 1987. ART neural networks are designed to allow the user to control the degree of similarity of patterns placed on the same cluster. However, since input patterns may differ in their level of detail (number of components that are non-zero), the relative similarity of the input pattern to the weight vector for a cluster unit, rather than the absolute difference between the vectors, is used. (A difference in one component is more significant in patterns that have very few nonzero components than it is in patterns with y few nonzero components than it is in patterns with many nonzero components). As the net is trained, each training pattern may be presented and then placed on a different cluster when it is presented later (due to change in the weights for the first cluster if it has learned other patterns in the meantime). A stable net will not return a pattern to a previous at different stages of training indicates an unstable net. Some nets achieve stability by gradually reducing the learning rate as the same set of training patterns is presented many times. However, this does not allow the net to learn readily a new pattern that is presented for the first time after a number of training epochs have already taken place. The ability of a net to respond to (learn) a new pattern equally well at any stage of learning is called plasticity. Adaptive resonance theory nets are designed to be both stable and plastic [33].

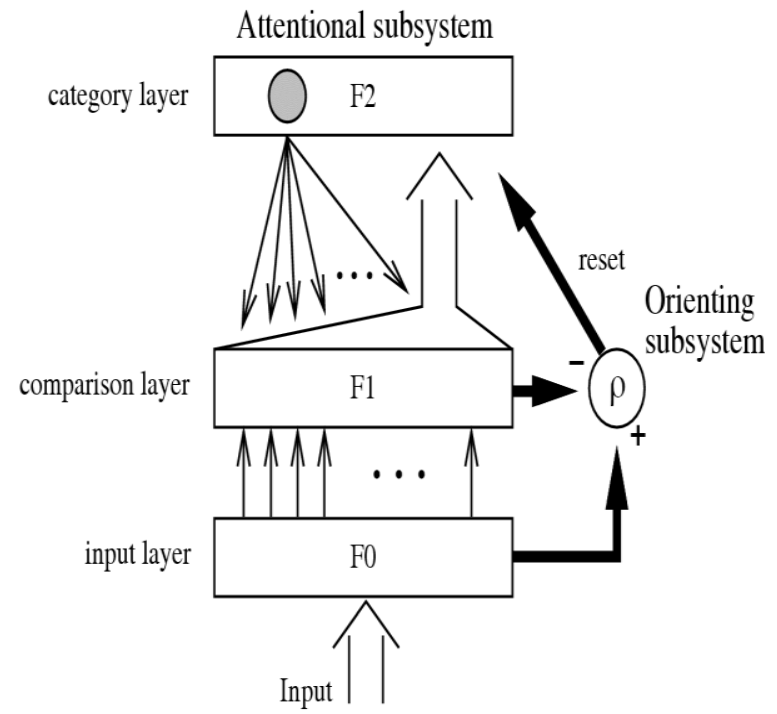

Fig. 4 Architecture of the ART network [30]

Neural-networks algorithms use simple computations, do not represent big burden to memory and the proposed modified ART models can be easily parameterized according to user needs for greater or lower level of details of the sensor data. The outputs of the ART neural networks can also be easily transformed into if-then decision rules understandable to humans [30].

\subsubsection{Fuzzy ART}

Fuzzy ART [11] model developed herein generalizes ARTl to be capable of learning stable recognition categories in response to both analog and binary input patterns. Fuzzy ART model has been incorporated into a Fuzzy ARTMAP architecture that can rapidly learns stable categorical mappings between analog or binary input and output vectors. Fuzzy ART incorporates the basic features of all ART systems, notably, pattern matching between bottom-up input and top-down learned prototype vectors. This matching process leads either to a resonant state that focuses attention and triggers stable prototype learning or to a self-regulating parallel memory search. If the search ends by selecting an established category, then the category's prototype may be refined to incorporate new information in the input pattern. If the search ends by selecting a previously untrained node, then learning of a new category takes place [57]. 
In [15] authors have presented an energy efficient solution based on wireless sensor networks for monitoring of the environment by traffic control. The algorithm is based on Fuzzy ART model of neural networks. Our system provides high dimensionality reduction when sending only the classified data and transferring only the new data in given time series. In this way, the system can be very energy efficient for monitoring of not frequent events.

\subsubsection{ART1}

ART1 [9] is designed to cluster binary input vectors, allowing for great variation in the number of nonzero components, and direct user control of the degree of similarity among patterns placed on the same cluster unit. The architecture of an ART1net consists of two fields of units- the $F_{1}$ units and the $\mathrm{F}_{2}$ (cluster) units-together with a reset unit to control the degree of similarity of patterns placed on the same cluster unit[33].This network learns clusters in an unsupervised mode. The novel property of the ARTl is the controlled discovery of clusters. In addition, it can accommodate new clusters without affecting the storage or recall capabilities for clusters already learned. The network produce clusters by itself; such clusters are identified in input data, and store the clustering information about patterns or features without a priori. Essentially the network "follows the leader" after it originates the first cluster with the first input pattern received. It then creates the second cluster if the distance of the second pattern exceeds a certain threshold; otherwise the pattern is clustered with the first cluster. This process of pattern inspection followed by either new cluster origination or acceptance of the pattern to the old cluster is the main step of ARTl neural network [57].

The authors in [52] have described that the performance of WSNs strongly depends on their network lifetime. They have observed that the improvement in network varies according to the network topology. ART1 is better than ART and the improvement in lifetime in ART1 is consistently around $45 \%$. The maximum network lifetime improvement is found to be $47 \%$. This effectively improves the bandwidth of the communication channel and also reduces the energy consumption.

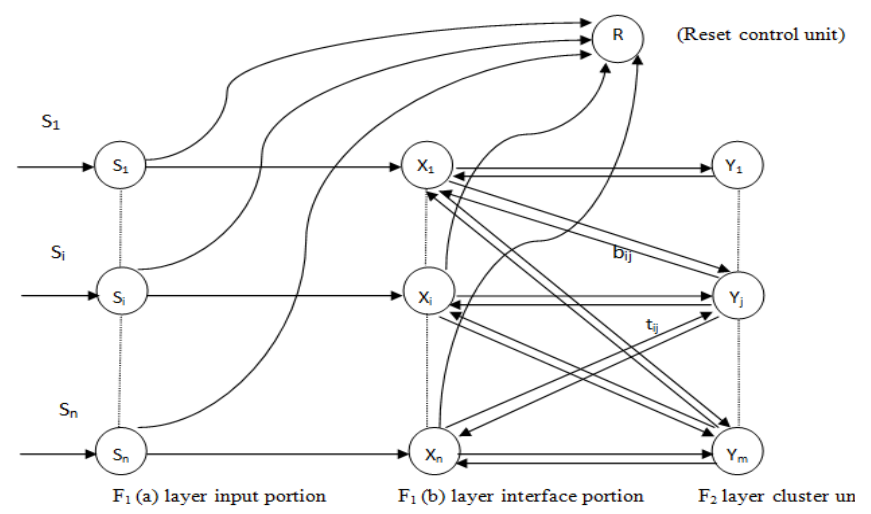

Fig.5 Basic Architecture of ART1 [58]

In [2], authors have focused on use of classification techniques using neural network to reduce the data traffic from the node and thereby reduce energy consumption. The sensor data is classified using ART1 Neural Network Model. Wireless sensor network populates distributed nodes. Directed diffusion routing protocol is implemented to carry out performance comparison. The paper discusses classification technique using ART1 neural network models. Lifetime improvement of the WSN is compared with and without classification using cooperative routing and diffusion routing.

In [58] paper, authors improve the network's lifetime through the mechanism including a minimum cluster head separation distance, an ART1 based cluster head election, a cluster head rotation system and load balancing cost functions. Experimental result implemented in MATLAB shows that ART1 neural network increases the network's lifetime by $60 \%$ when it is compared to the traditional LEACH algorithm. Consequently it enhances the quality of network.

\subsubsection{ART-2}

ART2 [10] is designed to perform for continuous-valued input vectors the same type of task as ART1 does for binary valued input vectors. The differences between ART1 and ART2 reflect the modifications needed to accommodate patterns with continuous-valued components. The more complex $F_{1}$ field of ART2 is necessary because continuous-valued input vectors may be arbitrarily close together. The $F_{1}$ field in the ART2 includes a combination of normalization and noise suppression, in addition to the comparison of bottom-up and top-down signals needed for the reset mechanism. There are two types of continuous-valued inputs for which ART2 may be used. The first might be called "noise binary" signals. These consist of patterns whose information is conveyed primarily by which components are "on" or "virtually off," rather than by the differences in the magnitude of the components that are positive. The equilibrium weights found by the fast learning mode are suitable for this type of data. However, it is not as easy to find equilibrium weights in ART2as it is for ART1, because the differential equations for the learning progresses [33].The scope of ART2 neural network is larger than others among ART theory. ART2 has many advantages. First, its training is self-organized and it has the capacity of studying without supervision. Second, it could identify patterns which have been studied before and adapt to new patterns. Third, it could identify the dynamic pattern [56].

In [22] authors have aimed at the severe energy and computing resource constraints of Wireless Sensor Network (WSN), based on rough set theory and ART2 network, a distributed data mining model for WSN is proposed. The input layer neuron and the first layer neuron are located in every cluster member, while the second layer neuron and the output layer neuron are located in every cluster head. The features of the training samples were extracted to build up the decision table; the rough set theory was applied to reduce the decision table. Finally, the reduced decision attributes were used to construct ART2 neural network classification data. Simulation results prove data dimension is reduced and data redundancy is eliminated after the raw-data is processed by data mining algorithm, and the communication traffic is decreased and the life of WSN is extended.

\subsubsection{Counter propagation networks}

Counterpropagation networks [20], [21] are multilayer networks based on a combination of input, clustering, and output layers. Counterpropagation nets can be used to compress data, to approximate functions, or to associate patterns. It approximates its training input vector pairs by adaptively constructing a look-up table. A large number of training data points can be compressed to a more manageable number of look-up table entries. If the training data represent 
functions values, the net will approximate a function.

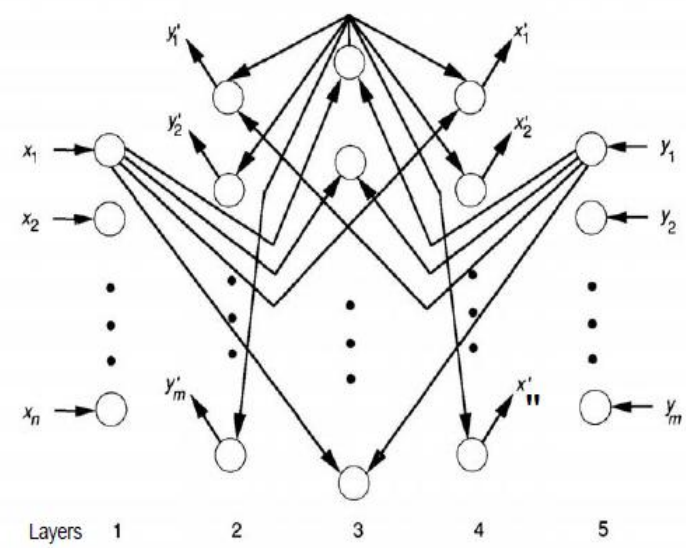

Fig.6 This splider like diagram of the counter propagation network has five layers: two input layers ( 1 and 5), one hidden layer (3), and two output layers ( 2 and 4 ).

A hetero-associative net is simply one interpretation of a function from a set of vectors (patterns) $x$ to a set vectors $y$. the accuracy of the approximation is determined by the number of entries in the look-up table, which equals the number of units in the cluster layer of the net. Counterpropagation nets are trained in two stages. During the first stage, the input vectors are clustered. During the second stage of training, the weights from the cluster units to the output units are adopted to produce the desired response. [33].

\subsubsection{Hopfield networks}

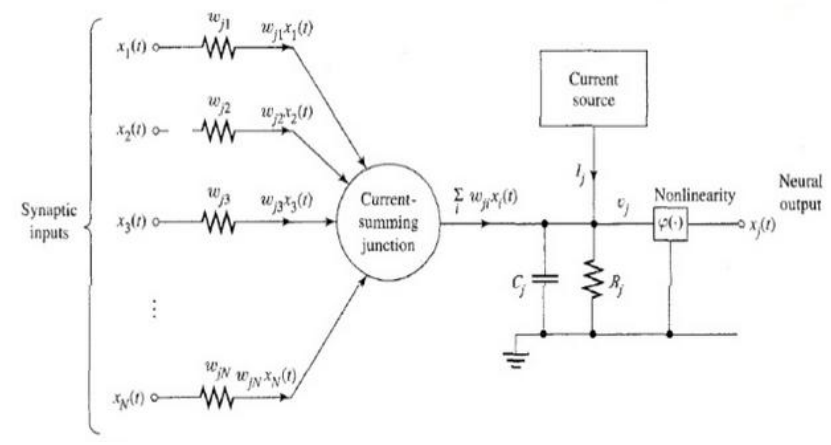

Fig.7 Hopfield neural network

Hopfield networks are constructed from artificial neurons. These artificial neurons have $\mathrm{N}$ inputs. With each input $i$ there is a weight wi associated. They also have an output. The state of the output is maintained, until the neuron is updated. Updating the neuron entails the following operations:

- The value of each input, $x i$ is determined and the weighted sum of all inputs, $\sum_{\mathrm{i}} \mathrm{w}_{\mathrm{i}} \mathrm{x}_{\mathrm{i}}$ is calculated.

- The output state of the neuron is set to +1 if the weighted input sum is larger or equal to 0 . It is set to -1 if the weighted input sum is smaller than 0 .

- A neuron retains its output state until it is updated again.

Formula:-

$$
\mathrm{o}=\left\{1: \sum_{\mathrm{i}} \mathrm{w}_{\mathrm{i}} \mathrm{x}_{\mathrm{i}} \geq 0 \text { or }-1: \sum_{\mathrm{i}} \mathrm{w}_{\mathrm{i}} \mathrm{x}_{\mathrm{i}} \leq 0\right\}
$$

A Hopfield network is a network of $\mathrm{N}$ such artificial neurons, which are fully connected. The connection weight from neuron $\mathrm{j}$ to neuron $\mathrm{i}$ is given by a number wij. The collection of all such numbers is represented by the weight matrix $\mathrm{W}$, whose components are wij. Now given the weight matrix and the updating rule for neurons the dynamics of the network is defined if we tell in which order we update the neurons. There are two ways of updating them:

- Asynchronous: one picks one neuron, calculates the weighted input sum and updates immediately. This can be done in a fixed order, or neurons can be picked at random, which is called asynchronous random updating.

- Synchronous: the weighted input sums of all neurons are calculated without updating the neurons. Then all neurons are set to their new value, according to the value of their weighted input sum.

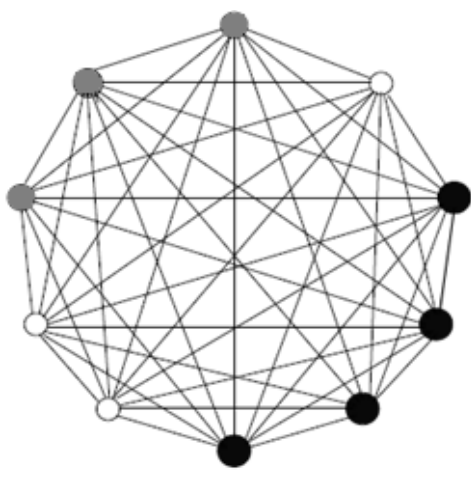

Fig.8 A Hopfield network as an autoassociator

Hopfield neural network has implemented for providing more secured communication in wireless sensor network. The authors have presented a key agreement scheme without the trusted third parties (TTP) by exploiting the special characteristics of Hopfield neural network. The main idea is to combine key agreement with the principle of convergence in Hopfield neural network. Experimental results shown that technique requires less memory and has lower communication overhead than the existing scheme [4].

\subsubsection{Kohonen self-organizing maps (SOM)}

Kohonen Self-Organizing Maps (or just Self-Organizing Maps, or or SOMs), are a type of neural network. They were developed in 1982 by Tuevo Kohonen, a professor emeritus of the Academy of Finland. Self-Organizing Maps are aptly named. "Self-Organizing" is because no supervision is required. SOMs learn by their own unsupervised competitive learning. "Maps" is because they attempt to map their weights to conform to the given input data. The nodes in a SOM network attempt to become like the inputs presented to them. In this sense, this is how they learn. They can also be called "Feature Maps", as in Self-Organizing Feature Maps. Retaining principle 'features' of the input data is a fundamental principle of SOMs, and one of the things that makes them so valuable. Specifically, the topological relationships between input data are preserved when mapped to a SOM network. Self-organizing feature maps (SOFM) learn to classify input vectors according to how they are grouped in the input space. They differ from competitive layers in that neighbouring neurons in the self-organizing map learn to recognize neighbouring sections of the input space. Thus, self-organizing maps learn both the distribution (as do competitive layers) and topology of the input vectors they are trained on.

The self-organizing neural networks described in this section, also called topology-preserving maps, assume a topological structure among the cluster units. This property is observed in 
the brain, but is not found in other artificial neural network. There are $m$ cluster units, arranged in a one- or twodimensional array; the input signals are $n$-tuples [29].

The weight vector for a cluster unit serve as an exemplar of input patterns associated with that cluster. During the selforganization process, the cluster unit whose weight vector matches the input pattern most closely is chosen as the winner. The winning unit and its neighboring units update their weights. The weight vectors of neighboring units are not, in general, close to the input pattern. For example, for a linear array of cluster units, the neighborhood of radius $\mathrm{R}$ around cluster unit $\mathrm{J}$ consists of all units $\mathrm{j}$ such that [33].

$$
\max (1, \mathrm{~J}-\mathrm{R}) \leq \mathrm{j} \leq \min (\mathrm{J}+\mathrm{R}, \mathrm{m}))
$$

\section{Architecture}

Each node in the SOM is mapped to neuron in the neural network. The architecture of SOM is shown in the "Fig.9". The neighborhood of the radii $\mathrm{R}=2,1$ and 0 are shown in the "Fig.10" for a rectangular grid and in "Fig. 11" for hexagonal grid. In each illustration, the winning unit is indicated by the symbol "\#" and the other units are denoted by "**". Note that each unit has eight nearest neighbors in the rectangular grid, but only six in the hexagonal grid. Winning units that are close to the edge of the grid will have some neighborhoods that have fewer units than that shown in the respective figure.

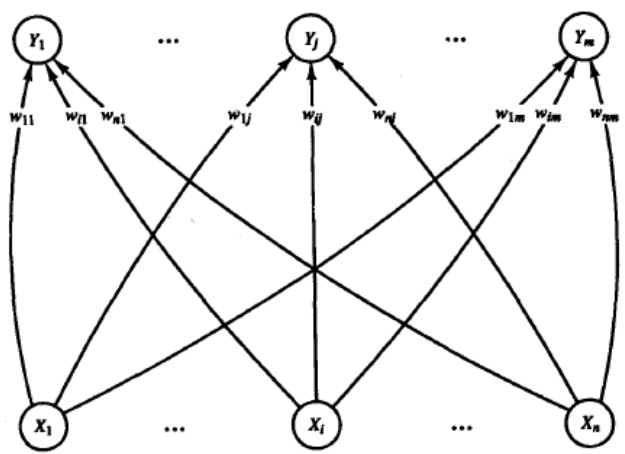

\section{Fig. 9 Kohonen self-organizing feature map}

Alternative structures are possible for reducing $\mathrm{R}$ and $\alpha$.The learning rate $\alpha$ is a slowly decreasing function of time or training epochs. Kohonen indicates that a linearly decreasing function is satisfactory for practical computations; a geometric decrease would produce similar results. The radius of the neighborhood around the cluster unit also decreases as the clustering process progresses.

Kohonen's Self Organizing Maps (SOMs) have been implementated in the field of wireless sensor network. Providing an efficient localization system is one of the most important goals to be pursued if an efficient utilization of sensor networks has to be addressed. A SOM is a particular neural network that learns to classify data without any supervision. In each sensor node, a SOM is implemented to evaluate the sensor node position, using a very little amount of storage and computing resources. In a scenario where thousands of sensor nodes are placed, this system evaluates the position of each sensor in a distributed manner, assuming a very little percentage of nodes knowing their actual position.

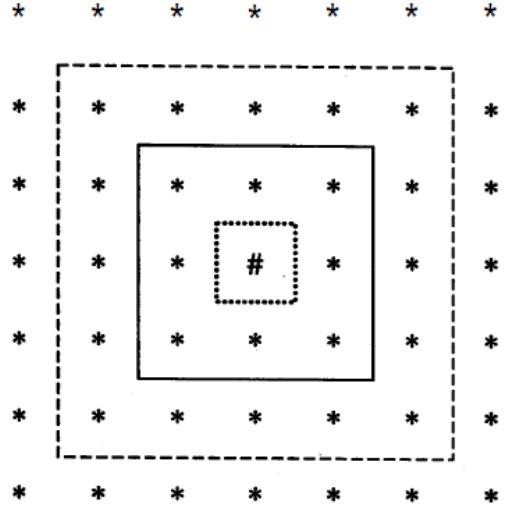

Fig.10 Neighborhood for rectangular grid

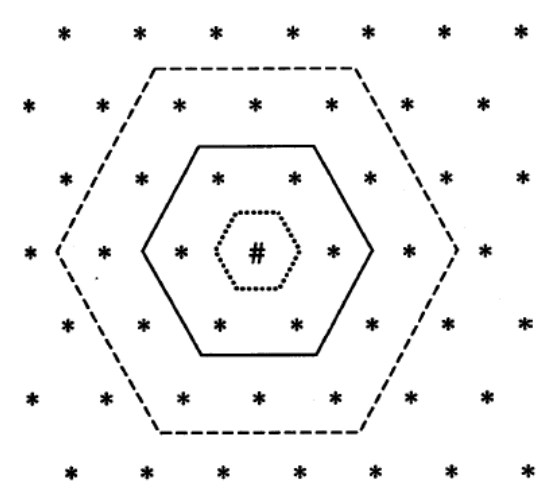

Fig.11 Neighborhoods for hexagonal grid

In [27] authors have discussed the main concern in Wireless Sensor Networks is that how to handle with their limited energy resources. The performance of Wireless Sensor Networks strongly depends on their lifetime. This paper presents a new centralized adaptive Energy Based Clustering protocol through the application of Self organizing map neural networks (called EBC-S) which can cluster sensor nodes, based on multi parameters; energy level and coordinates of sensor nodes. We applied some maximum energy nodes as weights of SOM map units; so that the nodes with higher energy attract the nearest nodes with lower energy levels. Simulation results and comparison with previous protocols (LEACH and LEA2C) prove that our new algorithm is able to extend the lifetime of the network

\subsection{Supervised Learning}

Supervised learning, at each instant of time, when the input is applied, the desired response of the neural network system is provided by the teacher. The distance between the actual and the desired output/response serves as an error measure; and is used to correct network parameters externally. The teacher may implement a reward-and-punishment scheme to adapt the network's weight matrix. For instance, in learning classifications of input patterns or situations with known responses, the error can be used to modify weights so that the error decreases. This mode of learning is very pervasive. Supervised learning rewards accurate classifications or associations and punishes those which yield inaccurate responses. The teacher estimates the negative error gradient direction and reduces the error accordingly. In many situations, the inputs, outputs and the computed gradient are deterministic, however, the minimization of error proceeds over all its random realizations. As a result, most supervised learning algorithms reduce to stochastic minimization of error in multi-dimensional weight space [57]. 


\subsubsection{Perceptron learning rule}

Perceptrons [42] perhaps the most far- reaching impact of any of the early neural networks. The percetron learning rule is more powerful learning rule then the hebb rule [33]. For the perceptron learning rule, the learning signal is the difference between the desired and actual neuron's response. Thus, learning [57] is supervised and the learning signal is given by

$$
\mathrm{r} \equiv \mathrm{d}_{\mathrm{i}}-\mathrm{o}_{\mathrm{i}}
$$

Where, $o_{i}$ is the actual output and $o_{i}=\operatorname{sgn}\left(w_{i}^{t} x\right)$, and $d_{i}$ is the desired response. Weight adjustments in this method, $\Delta \mathrm{w}_{\mathrm{i}}$ and $\Delta \mathrm{w}_{\mathrm{ij}}$, are obtained as follows

$$
\Delta \mathrm{w}_{\mathrm{i}}=\mathrm{c}\left[\mathrm{d}_{\mathrm{i}^{-}} \operatorname{sgn}\left(\mathrm{w}_{\mathrm{i}}^{\mathrm{t}} \mathrm{x}\right)\right] \mathrm{x}
$$

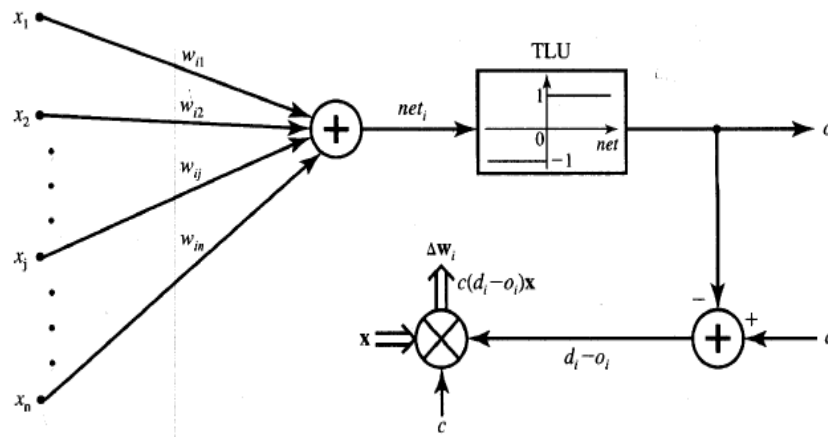

Fig. 12 Perceptron learning rule [57].

\subsubsection{Delta learning rule}

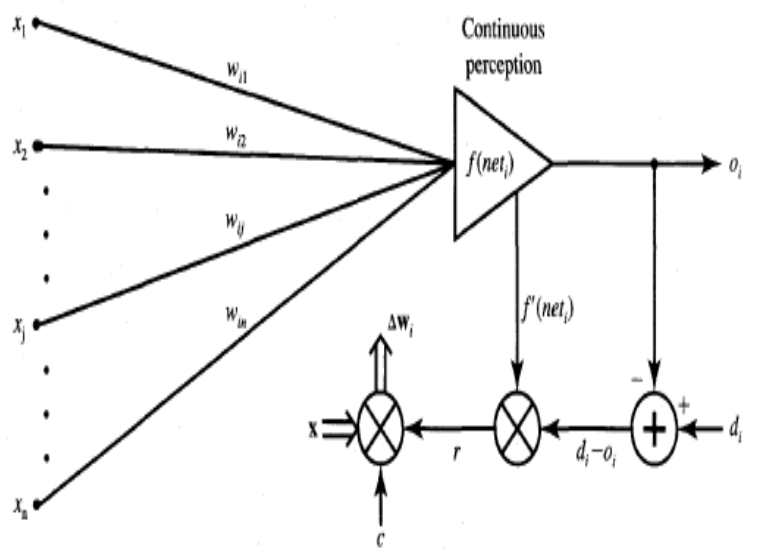

Fig. 13 Delta learning rule [57].

The delta learning rule is only valid for continuous activation functions and in the supervised training mode [57]. The learning signal for this rule is called delta and is defined as follows

$$
\mathrm{r} \equiv\left[\mathrm{d}_{\mathrm{i}}-f\left(\mathrm{w}_{\mathrm{i}}^{\mathrm{t}} \mathrm{x}\right)\right] f^{\prime}\left(\mathrm{w}_{\mathrm{i}}^{\mathrm{t}} \mathrm{x}\right)
$$

The term $\mathrm{f}^{\prime}\left(\mathrm{w}_{\mathrm{i}} \mathrm{x}\right)$ is the derivative of the activation function $\mathrm{f}$ (net) computed for net $=\mathrm{w}_{\mathrm{i}} \mathrm{x}$. This learning rule can be derived from the condition of least squared error between $o_{i}$ and $\mathrm{d}_{\mathrm{i}}$. Calculating the gradient vector with respect to $\mathrm{w}_{\mathrm{i}}$ of the squared error defined as

$$
\mathrm{E} \equiv \frac{1}{2}(d i-o i)^{2}
$$

For the single weight, the adjustment becomes

$$
\Delta \mathrm{w}_{\mathrm{i}}=\eta\left(\mathrm{d}_{\mathrm{i}}-\mathrm{o}_{\mathrm{i}}\right) f^{\prime}\left(\text { net }_{\mathrm{i}}\right) \mathrm{x}_{\mathrm{j}}, \text { for } \mathrm{j}=1,2, \ldots, \mathrm{n}
$$

\subsubsection{Error back propagation}

After an input pattern has been applied as a two- phase propagates-adapt cycle. After an input pattern has been applied as a stimulus to the first layer of network units, it is propagated through each upper layer unit an output is generated. This output pattern is then compared to the desired output, and an error signal is compared for each output unit. The error signals are then transmitted backward from the output layer to each node in the intermediate layer.

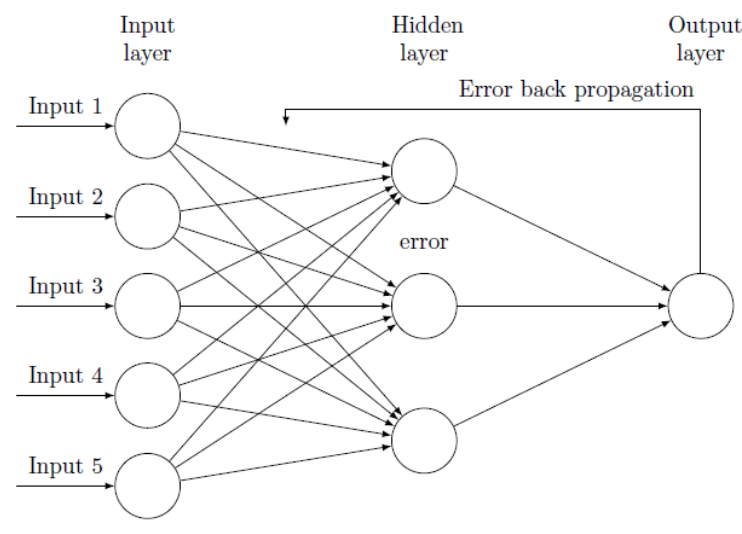

Fig. 14 Error back propagation

That contributes directly to the output. However, each unit in the intermediate layer receives only a portion of the total error signal, based roughly on the relative contribution the unit made to the original output. This process repeats, layer by layer, until each node in the network has received an error signal that describes its contribution to the total error. Based on the error signal received, connection weights are then updated by each unit to cause the network to converge toward a state that allows all the training patterns to be encoded. The significance of this process is that, as the network trains, the nodes in the intermediate layers organize themselves such that different nodes learn to recognize different features of the total input space. After training, when presented with an arbitrary input pattern that is noisy or incomplete, the units in the hidden layers of the network will respond with an active output if the new input contains a pattern that resembles the feature the individual units learned to recognize during training. Conversely, hidden layer units have a tendency to inhibit their output if the input pattern does not contain the feature that they were trained to recognize. As the signals propagate through the different layers in the network, the activity pattern present at the upper layer can be thought of as a pattern with features that can be recognized by units in the subsequent layer. The output pattern generated can be thought of as a feature map that provides an indication of the presence or absence of many different feature combinations at the input. The total effect of this behaviour is that the BPN provides an effective means of allowing a computer system to examine data patterns that may be incomplete or noisy, and to recognize subtle patterns from the partial input [33].

The authors in [43] have discussed accurate localization of nodes is one of the key issues of wireless sensor network (WSN). Considering the proportion of beacons in the network, localization algorithm based on sub-anchors and BP network is proposed in this paper to virtually increase the number of the anchors, and the method of finding appropriate subanchors is brought up by the virtual nodes leading to higher localization accuracy. Such algorithm utilizes not only the 
parallelism of neural networks but also the hops of the nodes, so that it is simple and easy to achieve in the hardware. The simulation shows the effectiveness of using virtual nodes to find sub-anchors, and indicates that sub-anchors and virtual nodes based on BP neural network could greatly improve the accuracy of the unknown nodes and reduce the costs of WSN.

\section{NEURAL NETWORK BASED ENERGY EFFICIENCY IN WIRELESS SENSOR NETWORKS}

The main concern in wireless sensor networks is how to handle with their limited energy resources. The performance of wireless sensor networks strongly depends on their lifetime. Neural Networks are not energy conservation methods and cannot independently help to conserve energy but they can help energy conservation methods as intelligent tools to work in more efficient, desirable and easier way. So the energy conservation methods are the same previous methods which can use neural network as a tool to better approach to their goals [37].

\subsection{Energy efficient path discovery}

The Self-Organizing Map (SOM) is an unsupervised neural network structure consists of neurons organized on a regular low dimensional grid [53].

In [48] authors proposed an intelligent method based on Self Organizing Map neural networks that optimize the routing in the terms of energy conservation and computation power of each node. This algorithm has been designed for a wireless sensor node called MODABER. The assumption is that every node has an importance due to its role in routing so that the nodes which are used more than other nodes in routing have more importance due to their positions. They defined a Network Life Time (NLT) parameter which is sum of the nodes importance in routing at time $t$ and the amount of energy consumption of node for routing. They used a selforganizing neural network to decide for every node containing the data packet and participate in routing or dropping the packet. The Self Organizing Map (SOM) learning algorithm is used for training of neural network.

As soon as a packet arrives, its feature vector will be extracted and this vector is sent to self organizing $\mathrm{NN}$ of that node as input. The goal is to maximize NLT parameter. After winning of node in competition against other nodes, it is allowed to send the packet and participate in routing. Otherwise it should drop the packet. Since the learning algorithms of SOMs generally obey from linear computations, they believe that this method can be efficient to wireless nodes due to their limited computation and energy powers [37].

SIR [7] is another QoS-driven SOM based routing protocol in which a SOM neural network is introduced in every node to manage the routes that data have to follow. They proved that the inclusion of AI techniques (e.g. neural networks) in wireless sensor networks is useful tools to improve network performances.

Usually a wireless sensor network life-time ends by having a single sensor node which uses all its power while other sensors have a significant amount of remaining power. The node which is in the routing path of many nodes to the base station is called a hotspot. In order to predict hotspots in a WSN, Authors in [24] defined a set of attributes for each sensor which were used as the inputs of our 3-layered back propagation neural network. These attributes belong to one wireless sensor node and by using them as the inputs of the neural network. They can predict the power level of the sensor at the end of WSN's lifetime. The predicted hotspots are then used in an Agent-based WSN route discovery and task management [37].

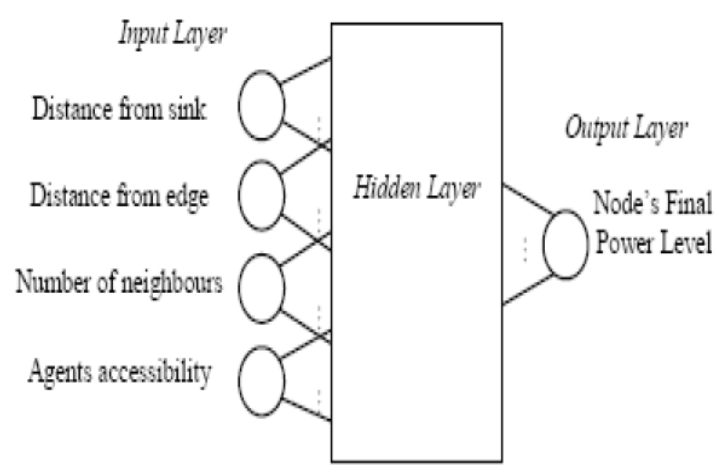

Fig. 15 Back Propagation neural network applied in [24] to predict the final power level of the node.

\subsection{Energy efficient node clustering}

WSN has been divided into clusters; the communication between nodes can be intra-cluster or inter-cluster. Intracluster communication comprises the message exchanges between the participating nodes and the $\mathrm{CH}$. Inter-cluster communications includes the transmission of messages between the $\mathrm{CHs}$ or between the $\mathrm{CH}$ and the BS. In all the cluster-based protocols we can identify three main phases during the clustering establishment process:

(a) Cluster head election,

(b) Cluster formation (set-up phase),

(c) Data transmission phase (steady-state phase) [13].

Cluster based routing are the most frequently used energy efficient routing protocols in Wireless Sensor Networks which avoid single gateway architecture through dividing of network nodes into several clusters while cluster head of each cluster play the role of a local base station[37].

The authors in [5] used Kohonen SOM neural networks for clustering and their analysis to study unpredictable behaviors of network parameters and applications. Clustering of sensor nodes using Kohonen Self Organizing Map (KSOM) is computed for various numbers of nodes by taking different parameters of sensor node such as direction, position, number of hops, energy levels, sensitivity, latency, etc.

SOM is an excellent tool for clustering of Wireless Sensor Networks because it is able to reduce dimensions of multidimensional input data and visualize the clusters into a map. Energy Based Clustering Self organizing map (EBC-S) [37] is a new topologic energy based clustering method through using Self Organizing Map neural networks which can efficiently extend the network lifetime and network coverage. 


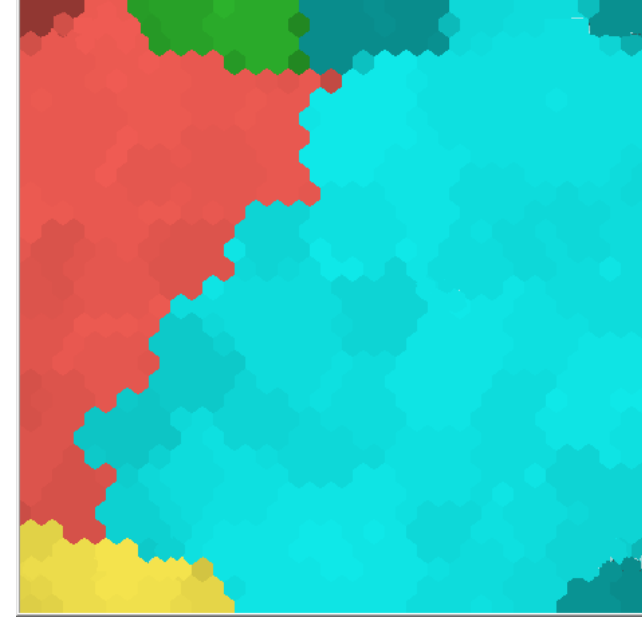

Fig.16 Clustering of sensor data using SOM neural network

In paper [60], authors have clustered the sensor environmental data taken temperature as parameter. There are four clusters are created through which one cluster has been selected for transfer of data towards the base station. The clusters are shown in following figure represented in different colors. Kohonen's Self-Organization Map (SOM) neural network algorithm has been efficiently used for data clustering; that learns to classify data without any supervision i.e. in unsupervised learning mode. Authors have analyzed and reduced the real data to make the network less bulky, communication gets faster as due to lager volume of data is get reduced, and end-to-end delay and power consumption of communication network also gets lowered.

\subsection{Cluster head selection}

The authors in [14] have proposed a new LEACH like routing protocol in which the election of Cluster Heads is done with SOM neural networks where SOM inputs are intended parameters for cluster heads. SOM cluster the nodes according to their cluster head qualities. However a minimum separation filter should be applied on SOM output then to ensure a minimum separation distance between selected $\mathrm{CHs}$ in figures 17 . The simulation Results show a $57 \%$ profit of this protocol over LEACH (in the terms of first dead time) [37].

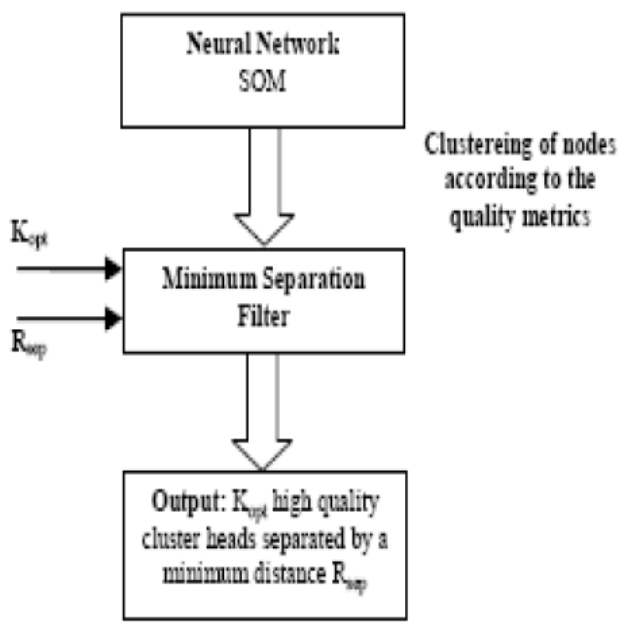

Fig. 17 General Operation of the SOM based cluster head selection mechanism [14].
The authors in [58] have proposed a new LEACH-ART routing protocol in which the election of Cluster Heads is done with ART1 neural networks. ART1 cluster the nodes according to their cluster head qualities. The simulation Results show a $60 \%$ profit of this protocol over LEACH (in the terms of first dead time) as shown in table II and table III as follows

TABLE I. Performance Metrics

\begin{tabular}{|c|c|c|c|}
\hline Metric & LEACH & $\begin{array}{c}\text { Algorithm with } \\
\text { ART1 neural } \\
\text { network (high } \\
\text { vigilance) }\end{array}$ & $\begin{array}{c}\text { Algorithm with } \\
\text { ART1 neural } \\
\text { network (low } \\
\text { vigilance) }\end{array}$ \\
\hline FND/seconds & 706 & 875 & 1078 \\
\hline HNA/seconds & 1000 & 1145 & 1115 \\
\hline $\begin{array}{c}\text { Transmitted } \\
\text { packets }\end{array}$ & 90504 & 101400 & 100200 \\
\hline Reclusters & 200 & 180 & 175 \\
\hline
\end{tabular}

TABLE II. IMPROVEMENT OF ART1 NEURAL NETWORK OVER LEACH

\begin{tabular}{|c|c|c|}
\hline Metric & $\begin{array}{c}\text { LEACH } \\
\text { Algorithm with ART1 } \\
\text { neural network (high } \\
\text { vigilance) }\end{array}$ & $\begin{array}{c}\text { LEACH } \\
\text { Algorithm with } \\
\text { ART1 neural } \\
\text { network (low } \\
\text { vigilance) }\end{array}$ \\
\hline FND/seconds & $35 \%$ & $60.34 \%$ \\
\hline HNA/seconds & $21.4 \%$ & $17.67 \%$ \\
\hline $\begin{array}{c}\text { Transmitted } \\
\text { packets }\end{array}$ & $11 \%$ & $9 \%$ \\
\hline Reclusters & $-3.5 \%$ & $-4.5 \%$ \\
\hline
\end{tabular}

\subsection{Data aggregation/fusion}

One of important issues of data fusion of WSNs is necessity of using an intelligent system which can fuse heterogeneous data obtained from different sources, accurately, automatically and efficiently. Data fusion can reduce the size of data. Moreover Even if the data had been affected by noise or intentional manipulating, data fusion method must be able to classify and identify the data. Sensor data fusion is a certain requirement of target detection and tracking applications in WSNs. One comprehensive survey study on sensor fusion approaches in target tracking is presented in [Smith and Singh (2006)]. One of the intelligent tools for data fusion is Neural Network [37].

A neural network method based on Hopfield structure proposed in [55] for this problem which always finds the optimal solution in 17.4 percent of the times and finds a way that approximate the proper solution in remained time.

One of the earliest applications of neural networks multisensor data fusion for identification was in [12] which applied a Back Propagation neural network. In a Back Propagation Neural Network, the data is given to the network and the difference between the input and output is calculated. Weights are changed to improve the result [37].

\subsection{Mobile data association}

The mobile sensor data association in target tracking is one of most important techniques for WSN. The main issue in data association tracking algorithms is to partition the sensor data into sets of observations produced by the same target, and the other one is to avoid the couple effect exists between the 
mobile sensors for the same target. Data Association Algorithms (DAAs) consist of three parts: acquiring, processing and combining. Mobile sensor tracking with DAA is a prerequisite step for mobile sensor surveillance systems over WSN deployment [17] [37].

Neural network approaches based on Hopfield Neural networks (HNN) have been proposed in [46] to solve this problem. HNNs which take weighted objective cost and constraints into an overall energy function are employed to combine with the neural network approaches to work out good tracking results. The difficulty for applying this method in DAA was that the determination of weight values was too difficult and it usually fell into irrational results [37].

In [17] the authors tried to take advantages of HNN so they improved the Competitive Hopfield Neural Networks (CHNN) algorithm which already had been applied in image processing applications. CHNN method can solve the above said problems by artfully managing of the weight updating function and the cost measurements. CHNN is an improved HNN in which a decision is made cooperatively [37].

The competitive updating scheme of weights can solve the problem of determination of weight values, guarantee the convergence into a stable solution and avoid from falling into irrational solutions [37].

\subsection{Context/Data classification}

Sensor nodes in an area usually form a sensing cluster and work together in a distributed and parallel way similarly to a layer of neurons. The data from all member sensors of a sensing cluster are from the same context but they are different because every sensor has a different point of view due to its different condition e.g. its position toward the event. Therefore these different data of cluster nodes have to be compressed and fused by in-network processing techniques [37].

In [38] a SOM neural network has been used for reduction and classification of similar patterns. They used SOM in hierarchical (cluster based) network architecture in which the nodes are organizes in several clusters with a cluster head or fusion centers. While reducing the amount of data to be transmitted, the SOM performs clustering of similar patterns. This characteristic enables the determination of relations between patterns which leads to their classification. This method can be applied in an event driven applications in where SOM can classify the event and increase the reliability of the decision [37].

In [35] researchers focused on using classification methods based on ART1 neural networks with the goal of reducing data traffic of node resulting in energy conservation. Sensor data which have too much redundancy, first, have to be classified by neural network in each node. Then, classified data were sent. In this way communication bandwidth increased efficiently [37].

\subsection{Data prediction}

New sensing methods with energy efficiency through prediction of sensor measurements have shown great ability in reducing communications in sensor networks. In these methods, sink node extract model of time series to predict local readings instead of communicate with sensor nodes and receive actual measurements which consume too much energy [37].

The authors in [49] used neural networks to schedule duty cycling of sensor nodes by event prediction. They proposed a neural method to decide which nodes and when have to be woken through prediction of the occurrence time of next event. The authors used neural network is a three layered Back Propagation which uses Morlet Wavelet transform at hidden layer. The nodes which are at deeper sleep, consumes more energy to wake up. So state of the nodes can be determined with prediction of time series of next event and by defining a threshold relative to remained energy of nodes and comparing of those with each other [37].

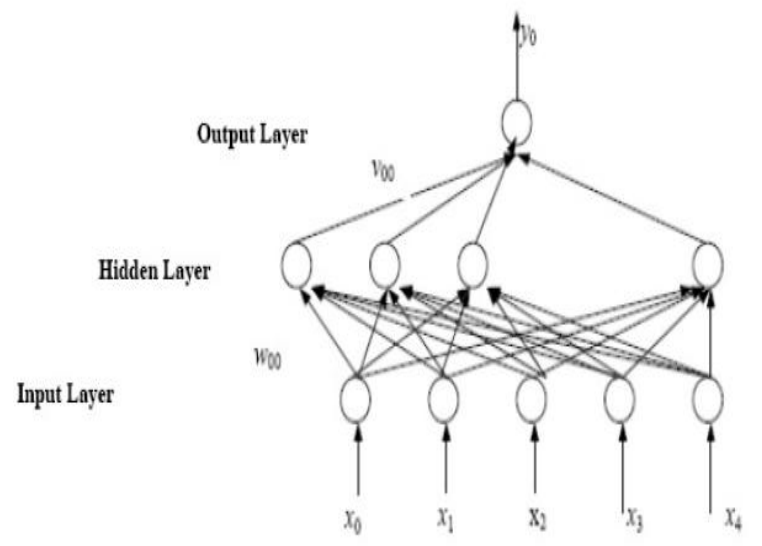

Fig. 17 The Wavelet Back Propagation Neural network Topology Structure [49]

4. CONCLUSION AND FUTURE SCOPE

In this paper we have shown a survey based on the use of neural network in WSN. Both the training methods of neural networks i.e. unsupervised and supervised, are implemented in wireless sensor network for enhancement of Quality of Services (QoS). QoS is basically focused on the end-to-end real-time and reliability, entirety sensor covered area in the wireless sensor network which is used to detect the events. Whenever the event occurs, it can be detected and transmitted rapidly into the WSN with low-energy consumption. The QoS mechanism should improve the network communication efficiency and reduce the energy consumption of sensor nodes to delay the network's survival time. Supervised learning neural networks algorithms like perceptron model, error back propagation work efficiently and effectively for the localization of sensor network and hence reduction in cost of sensor networks. On the other hand, unsupervised learning neural networks like ART, Fuzzy ART, ART1 and ART2 work at greater extent to improve the QoS mechanism which is fit for the WSN with limited resources; and it has the advantages such as less energy consumption, less computation amounts, less communication data etc. Self Organizing Map (SOM) neural network is very effective for the energy efficiency up-to a greater extent. SOM is also excellent in path discovery, node clustering, cluster head selection, data fusion, and context/data classification. Error back propagation (EBP) also gives better results in path discovery, data fusion and data prediction. ART1 is very efficient in context/data classification. Hopfield network works very well in mobile data association. However there is a good future scope in many neural network methods like winner-take-all, perceptron learning, ART, FUZZY ART, ART1, ART2 learning approaches to be used in energy conservation of wireless sensor network. So we can say that artificial neural networks play a very important role to improve the QoS parameters in WSNs. 


\section{REFERENCES}

[1] Akojwar, Sudhir G. and Patrikar, R.M. (2006), 'Real Time Classifier For Industrial Wireless Sensor Network Using Neural Networks with Wavelet Prerocessors', published in Industrial Technology, (ICIT ), IEEE International Conference, conference location: Mumbai, pp.512- 517.

[2] Akojwar, Sudhir G. and Patrikar, Rajendra M. (2008), 'Improving Life Time of Wireless Sensor Networks Using Neural Network Based Classification Techniques with Cooperative Routing ',published in international journal of communications, Vol. 2, Issue 1.

[3] Akojwar, Sudhir G. and Patrikar, Rajendra M.(2008), 'Classification Techniques Using Neural Networks and Clustering Architecture for Wireless Sensor Networks', Advances in Communication Systems and Electrical Engineering, publisher: Springer US, Volume 4, pp 3349.

[4] Ang Gao, Wei Wei and Zhixiao Wang (2010). 'Hopfieldassociation: Establishing a Shared Key in the Wireless Sensor Networks,' published in Second International Conference on Networks Security, Wireless Communications and Trusted Computing, pp. 70-73.

[5] Aslam N, Philips W, Robertson W, Siva Kumar SH, (2010), 'A multi-criterion optimization technique for energy efficient cluster formation in Wireless Sensor networks', Information Fusion, Elsevier, Volume 12, Issue 3 Pages 202-212.

[6] Barbancho J., Carlos Le'on, Javier Molina and Antonio Barbancho (2006), 'Giving neuron to sensor. QoS management in wireless sensor networks', Published in Emerging Technologies and Factory Automation (ETFA), IEEE Conference on 20-22 Sept. 2006, Conference Location: Prague, pp. $594-597$.

[7] Barbancho J, Leon C, Molina F.J, Barbancho A, (2007), 'Using artificial intelligence in routing scheme for wireless networks', Computer Communications, Elsevier, pp. 2802-2811.

[8] Cañete E., Chen J., Luque R.Marcos and Rubio Bartolome'(2012),'NeuralSens: A neural network based framework to allow dynamic adaptation in wireless sensor and actor networks', Journal of Network and Computer Applications, 2012, pp. 382-393.

[9] Carpenter, Gail A., \& Grossberg, S.(1987a), 'A Massively Parallel Architecture for a Self-Organinzing Neural Pattern Recognition Machine.', Computer Vision, Graphics, and Image Processing, 37:54-115.

[10] Carpenter, Gail A., \& Grossberg, S.(1987b), 'ART2: Self-organization of Stable Category Recognition Codes for Analog Input Patterns.', Applied Optics, 26:4919-4930, Reprinted in Anderson, Pellionisz, \& Rosenfeld(1990), pp. 151-162.

[11] Carpenter, Gail A., Grossberg, S. and Rosen, David B. (1991), 'Fuzzy ART: Fast Stable Learning and Categorization of Analog Patterns by an Adaptive Resonance System', Science Direct, Neural Networks, Vol. 4, pp. 759-771.
[12] Chaudhuri, S.P. and Das, S. (1990), 'Neural Networks for Data Fusion', Published in Systems Engineering, IEEE International Conference, pp.327-330

[13] Chunjuan Wei, Junjie Yang, Yanjie Gao and Zhimei Zhang (2011), 'Cluster-based Routing Protocols in Wireless Sensor Networks: A Survey', International Conference on Computer Science and Network Technology, IEEE, pp. 1659-1663.

[14] Cordina M, Debono C.J. (2008), 'Increasing Wireless Sensor Network Lifetime through the Application of SOM neural networks', ISCCSP, IEEE, pp. 467-471.

[15] Danco Davcev and Stojanco Gancev (2009),' Monitoring of environment by energy efficient usage of Wireless Sensor Networks, Information Technologies in Environmental Engineering', Proceedings of the $4^{\text {th }}$ International ICSC Symposium Thessaloniki, Greece, Publisher: Springer Berlin Heidelberg, pp. 229-237.

[16] Enami, N, Askari Moghadam R. (2010), 'Energy Based Clustering Self Organizing Map Protocol for Extending Wireless Sensor Networks Lifetime and Coverage', Canadian Journal on Multimedia and Wireless networks, AM Publishers, Vol.1, No.4, August 2010, pp.42-54.

[17] Feng, X., Xu, Z.H. (2009), 'A Neural Data Fusion Algorithm for Wireless Sensor Networks', Pacific-Asia Conference on Circuits, Communications and Systems, pp. 54-57.

[18] Fu Lin, DengYi Zhang and WenHai Li (2011), 'Research on Quality of Service in Wireless Sensor Networks', published in Software Engineering and Service Science (ICSESS), IEEE 2nd International Conference, conference location: Beijing, pp. 312-315.

[19] Hebb, D. O. (1949). The organization of Beahviour, New York: John Wiley \& Sons. Introduction and Chapter 4,"the first stage of perception: growth of assembly,"pp xi-xix, 60-78.

[20] Hecht-Nielsen, R. (1987a), 'Counterpropagation Networks’, Applied Optics, 26(23), pp. 4079-4984.

[21] Hecht-Nielsen, R. (1987b), 'Counterpropagation Networks', IEEE International conference on Neural Networks II., pp. 19-32.

[22] Hong Yuehua, Xu Shuang and Wu Huajian (2010), 'Study on Distributed Data Mining Model in Wireless Sensor Networks', published in Intelligent Computing and Integrated Systems (ICISS), International Conference, conference location: Guilin, pp. 866-869.

[23] Hortos, W. S. (2012), 'Effects Of Energy Harvesting on Quality-of-Service in Transient Wireless Sensor Networks', published in military Communications Conference, conference location : Orlando, FL, pp. 1 9.

[24] Hosseingholizadeh Ahmad and Abhari Abdolreza (2009), 'A neural network approach for Wireless sensor network power management'.

[25] Jun Zheng, Abbas Jamalipour, (2009), 'Wireless Sensor Networks: A Networking Perspective', published by John Wiley \& Sons, Inc., Hoboken, New Jersey. 
[26] Karthikeyan, B., Gopal, S. and Venkatesh, S. (2006), 'ART2 - an unsupervised neural network for PD pattern recognition and classification', Expert Systems with Applications, pp. 345-350.

[27] Kashani, M.A.A. and Hassan Ziafat (2011), 'A method for Reduction of Energy Consumption in Wireless Sensor Network with using Neural Networks', published in Computer Sciences and Convergence Information Technology (ICCIT), 2011 6th International Conference on Nov. 29, 2011-Dec. 1 ,2011, conference location: Seogwipo, pp. 476 - 481.

[28] Kay, J and Frolik, J.(2004), 'Quality of Service Analysis and Control for Wireless Sensor Networks', published in Mobile Ad-hoc and Sensor Systems, IEEE International Conference ,pp. 359-368.

[29] Kohonen, T. (1989). Self-organization and Associative Memory, $3^{\text {rd }}$ ed. Berlin:Springer Verlag.

[30] Kulakov A. , Danco Davcev and Goran Trajkovski (2005), 'Implementing artificial neural networks in wireless sensor networks', published in Advances in Wired and Wireless Communication, 2005 IEEE/Sarnoff Symposium on 18-19 April 2005, Conference location Princeton, NJ, pp. 94 - 97.

[31] Kulkarni, R.V, Forster, A. ; Venayagamoorthy, G.K.(2011), 'Computational Intelligence in Wireless Sensor Networks: A Survey', IEEE Communications Surveys \& Tutorials, VOL. 13, NO. 1, FIRST QUARTER 2011, pp. 68-96.

[32] Larios, D.F., Barbancho, J., Rodrı'guez, G., Sevillano, J.L., Molina, F.J. and Leo' n, C. (2012), 'Energy efficient wireless sensor network communications based on computational intelligent data fusion for environmental monitoring', published in Communications, IET, pp. 2189-2197.

[33] Laurene Fausett(1994), 'Fundamentals of Neural Networks , Architecutre, Algorithms and Applications', published by arrangement with Pearson Education, Inc. and Dorling Kindersley Publishing Inc.

[34] Luca Paladina, Maurizio Paone, Giuseppe Jellamo and Antonio Puliafito (2007), ' SelfOrganizing Maps for Distributed Localization in Wireless Sensor Networks', Published in Computers and Communications, (ISCC) 12th IEEE Symposium, pp. $1113-1118$.

[35] Mohamed, W.K., Mirza, O., Kawtharani, J. (2009), 'BARC: A Battery Aware Reliable Clustering algorithm for sensor networks', Journal of Network and Compute Applications 32(6), pp.1183-1193.

[36] Neeraj Kumar, Manoj Kumar and R.B. Patel (2009), 'Neural Network Based Energy Efficient Clustering and Routing in Wireless Sensor Networks', published in Networks and Communications, 2009, NETCOM '09, First International conference on date of 27-29 Dec. 2009, conference location: Chennai, pp. $34-39$.

[37] Neda Enami, Reza Askari Moghadam, Kourosh Dadashtabar \& Mojtaba Hoseini (2010), 'Neural Network Based Energy Efficiency in Wireless Sensor Networks: A Survey,' International Journal of Computer Science \& Engineering Survey (IJCSES), Vol.1, No.1, pp. 39-55.
[38] Oldewurtel, Frank and Mahonen, Petri, (2006), 'Neural Wireless Sensor Networks', International Conference on Systems and Networks Communications, ICSNC, pp. $28-28$.

[39] Radi M., Behnam Dezfouli, Shukor Abd Razak, Kamalrulnizam and Abu Bakar (2010), 'LIEMRO: A Low-Interference Energy-Efficient Multipath Routing Protocol for Improving QoS in Event-Based Wireless Sensor Networks', Published in Sensor Technologies and Applications (SENSORCOMM), Fourth International Conference, pp. 551-557.

[40] Rajan, P and Rajan, S.V. (2011), 'Network Supporting Multilayered Quality of Service Routing in Wireless Sensor Networks', published in Emerging Trends in Electrical and Computer Technology (ICETECT), International Conference, Conference Location : Tamil Nadu, pp. 1016 - 1025

[41] Reza Tati, Fariborz Ahmadi, Rahim Rashidy and Faroog Ashkoti (2009), 'Designing and simulation of a distributed algorithm for Quality of service in wireless sensor networks', published in Application of Information and Communication Technologies, AICT 2009. International Conference, conference location: Baku, pp.1-5.

[42] Rosenblatt, F. (1958), The Perceptron: A Probabilitistic Model for Informatioon Storage and Organization in the brain." Psychological Review, Vol 65(6), Nov 1958, 386-408.

[43] Runjie LIU, Kai SUN and Jinyuan SHEN (2010), 'BP localization algorithm based on virtual nodes in wireless sensor network', published in Wireless Communications networking and Mobile Computing (WiCOM), 6th International Conference on Sept. 2010, Conference Location: Chengdu, pp. $1-4$.

[44] Ruogu Zhou, Guoliang Xing, Xunteng Xu, Jianping Wang and Lin Gu (2013), 'WizNet: A ZigBee-based Sensor System for Distributed Wireless LAN Performance Monitoring', IEEE International Conference on Pervasive Computing and Communications, conference location: San Diego, CA, pp. 123-131.

[45] S. Haykin (1994),'Neural Networks: A Comprehensive Foundation', Prentice Hall, Prentice Hall PTR Upper Saddle River, NJ, USA.

[46] Sengupta, D., Iltis, R.A. (1989), 'Neural solution to Multitarget Tracking Data Association Problem', IEEE Trans. Aerosp. Electron. Syst. 25, pp.86-108.

[47] Serrano-Gotarrdeona, T. and Bernab'e LinaresBarranco (1997), 'An ART1 Microchip and Its Use in Multi-ART1 Systems', Published in Neural Networks, IEEE Transactions, vol. 8, issue no.5, pp. 1184 - 1194.

[48] Shahbazi, H., Araghizadeh, M.A., Dalvi, M., (2008), 'Minimum Power Intelligent Routing In Wireless Sensors Networks Using Self Organizing Neural Networks', IEEE International Symposium on Telecommunications, pp. 354-358.

[49] Shen, Y., Guo, B., (2008), 'Wavelet Neural Network Approach for Dynamic Power Management in Wireless Sensor Networks', International Conference on 
Embedded Software and Systems (ICESS2008), pp. $376-381$.

[50] Stojanco Gancev and Danco Davcev (2011), 'Monitoring Wireless Sensor Network System Based on Classification of Adopted Supervised Growing Neural Gas Algorithm', ICT Innovations, Communications in Computer and Information Science Volume 83, Publisher: Springer Berlin Heidelberg,

[51] Smith, D., Singh, S. (2006), 'Approaches to Multisensor Data Fusion in Target Tracking: A Survey', IEEE transactions on knowledge and data engineering, Vol. 18, issue no. 12, pp.1696-1710.

[52] Subhai, C.P., Malarkan, S. and Vaithinathan, K.; (2013), 'A Survey on Energy Efficient Neural Network Based Clustering Models In Wireless Sensor Networks', published in Emerging Trends in VLSI, Embedded System, Nano Electronics and Telecommunication System (ICEVENT), International Conference, Conference Location: Tiruvannamalai, pp. $1-6$.

[53] Vesanto J, Alhoniemi E. (2000), 'Clustering of Self Organizing Map,' IEEE Transactions on Neural Networks, Vol. 11, No. 3, pp. 586-600.

[54] Wen-Tsai Sung, Yu-Feng Liu, Jui-Hi Chen and ChiaHao Chen (2010), 'Enhance the Efficient of WSN data fusion by Neural Networks Training Process', published in Computer Communication Control and Automation (3CA), 2010 International Symposium on Vol. 2, date of conference: 5-7 May 2010, conference location :Tainan, pp. 373 - 376.

[55] Winter, M. and Favier, G. (1999), 'A Neural Network for Data Association', IEEE Int'l Conf. Acoustics, Speech, and Signal Processing, vol. 2, pp. 1041-1044.
[56] Xiujiang, Lv, Guangshun Yao, Yan Zhao, Qiwen Zhang, Yu'e Li and Ning Wanget (2006), 'An Improved Architecture Based on Typical ART-2 Neural Network', published in Systems and Control in Aerospace and Astronautics (ISSCAA), 1st International Symposium, conference location: Harbin, pp. 1119-1121.

[57] Zurada, Jacek M. (1999), 'Introduction to artificial neural systems', published by West Publishing Company, printed in United States of America.

[58] Mohit Mittal and Krishan Kumar, "Network lifetime enhancement of homogeneous sensor network using ART1 neural network", Sixth IEEE International conference on computational Intelligence and Communication Networks, 2014, pp. 472-475.

[59] Krishan Kumar, "Self-Organizing Map (SOM) Neural Networks for Air Space Sectoring, "Sixth IEEE International Conference on Computational Intelligence and Communication Networks (CICN), 2014, pp. 10961100.

[60] M. Mittal and K. Kumar, "Energy Efficient Homogeneous Wireless Sensor Network Using SelfOrganizing Map (SOM) Neural Networks," African Journal of Computing \& ICT Vol 8. No. 1, 2015, pp. 179-184.

[61] Dr. Krishan Kumar, "ART1 neural networks for air space sectoring," International Journal of Computer Applications, pp. 20-24, Jan. 2012.

[62] K. Kumar, R. Singh, Z. Khan, A. Indian, “Air Traffic Runway Allocation Problem Using ARTMAP (ART1)," Ubiquitous Computing and Communication Journal (UBICC), vol. 3, No 3, Jul. 2008. 